\title{
TeV Blazars as the Sources of Ultra High Energy Cosmic Rays
}

\author{
Bingkai Zhang1*, Xiaoyun Zhao', Zhen Cao² \\ ${ }^{1}$ Department of Physics, Fuyang Normal College, Fuyang, China \\ ${ }^{2}$ Institute of High Energy Physics, Chinese Academy of Sciences, Beijing, China \\ Email: ${ }^{*}$ zhangbk@mail.ihep.ac.cn
}

Received 26 June 2014; revised 25 July 2014; accepted 20 August 2014

Copyright (C) 2014 by authors and Scientific Research Publishing Inc.

This work is licensed under the Creative Commons Attribution International License (CC BY).

http://creativecommons.org/licenses/by/4.0/

(c) (i) Open Access

\begin{abstract}
The origin of ultra high energy cosmic rays (UHECRs) is still an open question in astroparticle physics. TeV blazars are a small group of active galactic nuclei (AGNs). They all have been observed in $\mathrm{TeV}$ gamma ray band, and show violent variabilities in flux at all wavelengths. So it is believed that they have abilities to produce UHECRs. To judge whether the TeV blazars can be the candidates of the origin of UHECRs, we collect the information of emission region of $38 \mathrm{TeV}$ blazars, and estimate the maximum energy that the charged particle can be accelerated there. The results show that $\mathrm{TeV}$ blazars have abilities to accelerate cosmic rays to the energy above $10^{18} \mathrm{eV}$, some even higher than $10^{20} \mathrm{eV}$, and they may be the sources of UHECRs.
\end{abstract}

\section{Keywords}

\section{BL Lacertae Objects, TeV Blazars, UHECRs}

\section{Introduction}

The earth's atmosphere is being constantly bombarded by elementary particles from outer space, historically called cosmic rays. Their discovery dates back to the beginning of the 20th century and they have been continuously studied by particle physicists and astrophysicists. The spectrum of cosmic rays extends smoothly over 11 orders of magnitude, from less than $10^{9} \mathrm{eV}$ to over $10^{20} \mathrm{eV}$. Over this large energy range the spectrum falls off approximately by an $\mathrm{E}^{-3}$ power law. Accurate measurement shows that the spectrum is structured with four noticeable features: the knee is around $3-5 \times 10^{15} \mathrm{eV}$, the second knee is at $4 \times 10^{18} \mathrm{eV}$ [1]-[3], the ankle is near $3 \times 10^{18} \mathrm{eV}$ [3] [4], GZK cutoff is above $4-5 \times 10^{19} \mathrm{eV}$ [5] [6] which has been observed independently by HiRes and Auger experiments [4] [7]. At the lower energies this flux is high enough for direct satellite or bal-

${ }^{*}$ Corresponding author. 
loon measurement. While at the highest energies, the flux is so low that an extremely large aperture must be used in the study of cosmic rays.

The sources of cosmic rays are still unknown. It is probable that for energies up to and possibly slightly beyond the knee, the observed flux can be accounted by acceleration in supernova remnants. The ankle in the spectrum may mean a change from galactic to extragalactic sources, especially as it occurs at certain energy where cosmic ray protons can no longer be confined in the galactic disk. It can be interpreted as the transition between the galactic and extragalactic components [8] or the result of pair production by extragalactic protons after the interaction with photons of the cosmic microwave background radiation (CMBR) during propagation [9] [10].

Ultra high energy cosmic rays (UHECRs) were defined as those cosmic rays with energies above $10^{18} \mathrm{eV}[11]$. Since the first evidence of a cosmic ray primary with energy of $10^{20} \mathrm{eV}$ was reported [12], a succession of detectors, such as AGASA (the Akeno Giant Air Shower Array) [13], HiRes (the High Resolution Fly's Eye) [14], Auger (the Pierre Auger Observatory) [15], and TA (the Telescope Array) [16], have been constructed to try to determine the energy spectrum, the primary composition, and the arrival direction anisotropy of UHECRs. Now the origin of UHECRs is still an open question in astrophysics. As yet, no astronomical sources have been identified to be the sources of UHECRs. How and where these particles gain their remarkable energies have not been well understood. Linsley noted that nothing in our galaxy was thought to be capable of accelerating charged particles to such energies [12]. Given the strength of galactic magnetic fields and the lack of correlations with the galactic plane, the highest energy cosmic rays are likely to originate in extragalactic sources. Current observations show that the spectrum is consistent with an origin in extragalactic astrophysical sources.

It is generally believed that astrophysical sites such as neutron stars (or magnetars), gamma-ray bursts and active galactic nuclei may be reasonable candidates for UHECR sources (see [17] [18] and references therein). Active galactic nuclei (AGNs) are one of the best candidates for UHECR sources, as far as acceleration and energetics is concerned [19]. TeV blazars (see next section) are a subclass of AGNs characterized by rapid and large variability at all frequencies. Thus, TeV blazars are more likely to be the origin of UHECRs. However, it is difficult to directly connect UHECRs to blazars due to the sparse UHECR events and the changes of the arrival direction of UHECRs by intergalactic magnetic field. To judge whether TeV blazars can be candidates of UHECRs, the key is to judge whether the charged particles in the sources can be accelerated to ultra high energy. The most straightforward mechanism for accelerating a charged particle is placing it in an electromagnetic field. The interaction of a charged particle with the electric field will boost the particle's energy while the interaction with the magnetic field will confine the particle. The gyroradius of the particle is dependent on its charge and energy, so at some point the particle will get enough energy to escape the acceleration region. The purpose of this paper is to estimate the maximum energy that the charged particles can be accelerated according to the size of the emission region and the strength of the magnetic field, and then determine whether $\mathrm{TeV}$ blazars can be the candidates of UHECR source. The paper is organized as following: in Section $2 \mathrm{TeV}$, blazars are introduced, in Section 3 the maximum energies are estimated, then in Section 4 the discussion is given and in Section 5 conclusions are drawn.

\section{TeV Blazars}

Blazars are a subclass of AGNs with a relativistic jet pointed close to the line of sight of the observer [20]. They have been observed at all wavelengths from radio to high energy gamma rays, and they show variability in flux at all wavelengths as well as spectral shape on time scales ranging from months to a few minutes. Blazars are divided into flat spectrum radio quasars (FSRQs) and BL Lacertae objects (BL Lacs). Since the first detection of $\mathrm{TeV}$ gamma rays from Mkn 421 with the Whipple imaging atmospheric Cherenkov telescopes [21], blazars have become one of the most interesting class of object for VHE gamma-ray astronomy. For the TeV emission is the most prominent feature in those sources, they are called TeV blazars, which constitutes a relatively small group of sources within the blazar family. The gamma-ray emission of most blazars peaks in the MeV-GeV range. Moreover, TeV gamma rays are efficiently absorbed by the extragalactic background light (EBL). This in addition limits the number of observed $\mathrm{TeV}$ blazars. Nowadays, about 50 blazars have been detected in the $\mathrm{TeV}$ gamma-ray band. These sources locate both in the northern and in the southern hemisphere.

The electromagnetic (EM) radiation from blazar is predominantly non-thermal, and the spectral energy distributions (SEDs) of blazars are characterized by two broad peaks. The low energy peak in the SED is located 
between infrared to X-ray energies, and the mechanism is almost certainly synchrotron emission from a relativistic distribution of electrons. According to the frequency of the low energy peak, BL Lacertae objects are further subdivided into low-, intermediate- and high-frequency peaked BL Lac objects, and they are abbreviated as LBL, IBL and HBL, respectively. The high energy peak is located gamma-ray energies. However, the origin of the high energy peak is still a matter of debate. There are several models to explain the high energy emission based on either leptonic or hadronic interactions. Under the assumption of leptonic models, high energy emission is explained via inverse Compton scattering of soft target photons. The target photons may come from synchrotron emission by the same population of electrons which produce the low energy bump in synchrotron self Compton (SSC) model or from the external photons to the jet in external Compton (EC) models. These external photons can be the accretion disk photons or the accretion disk photons reprocessed by broad line region (BLR) clouds or the infrared radiation from the dusty torus. The hadronic models explain the high energy emission as an outcome of the synchrotron proton emission and proton-photon interactions with the synchrotron photons (see [22] and references therein).

Simultaneous observations of blazars across multiple wavelengths have been made to construct the spectral energy distributions (SEDs). Some authors shed light on the VHE emission mechanisms of these TeV sources according to their SEDs and get some information of emission regions. We collect the magnetic field $\mathrm{B}$, linear size $R$ and Doppler factor $\Gamma$ of $38 \mathrm{TeV}$ blazars. Since different models or different SED data give different values of these parameters, we calculate their mean values and list them in Table 1 . The first column is for the object name, the second is for the red shift, the third is for the type, the fourth is for the magnetic field $\mathrm{B}$, the fifth is for the emission size $R$, the sixth is for the Doppler factor $\Gamma$ and the last is for the reference.

\section{Estimation of Maximum Energy}

There exist several theories describing mechanisms capable of producing particles above $10^{18} \mathrm{eV}$. These theories may be divided into two major classes: top-down decay scenario and bottom-up acceleration scenario [23]. Bottom-up acceleration describes processes that take a particle, such as a proton, and accelerate it to a very high energy. The model is the more conventional hypothesis but struggles to reach the highest energies observed. For bottom-up acceleration to work, the source must provide either a very large acceleration gradient or provide acceleration over a very large distance. Top-down mechanisms produce the particles locally using the decay or interaction of exotic particles such as the decay of a super heavy relic particle from the big bang or the interaction of a magnetic monopole and anti-monopole. In this model cosmic rays begin at energies at and preferably well above those observed, so top-down models can explain the existence of UHECR, while they have a more difficult time explaining the flux. Additionally, the models require the production of existence of very exotic particles. At present, no theory has yet to produce a conclusive mechanism nor has experiment been able to trace these particles to any conclusive source.

In the bottom-up models, irrespective of the details of the acceleration mechanism, the basic requirement that cosmic ray accelerators must meet was summarized by Hillas [24]. Charged particles will escape from an acceleration region when their gyroradii increase beyond the size of the acceleration region. Thus the size and magnetic field strength associated with an astrophysical acceleration site determine the maximum theoretical acceleration energy Emax for a charged particle. There are indications that jets, powered by super-massive black holes at the center of active galaxies, are cosmic ray accelerators. The cosmic ray acceleration process is assumed to occur within a relativistic blob of plasma moving along the jet with Lorentz factor $\Gamma \sim 10^{1.5}$ [25]. They produce photons of TeV energy, possibly higher, and may be the enigmatic source of the highest energy cosmic rays [26]. As acceleration site of charged particles, jets of AGN have the advantage that acceleration on the jet frame could have maximum energy smaller that these of the observed by $1 / \Gamma$ [27]. Thus, one can calculate the maximum theoretical acceleration energy [24] [28], that does not include any efficiency factor,

$$
\frac{E_{\max }}{10^{15} \mathrm{eV}} \leq \Gamma Z \mathrm{e}\left(\frac{B}{\mu \mathrm{G}}\right)\left(\frac{B}{\mathrm{pc}}\right)
$$

where $\Gamma$ is the Lorentz factor of the shock matter, $Z$ is the charge of the nucleus, $B$ is the strength of magnetic field, and $\mathrm{R}$ is the radius of the emission region.

In the jets of $\mathrm{TeV}$ blazars, the maximum energy of a particle with charge $Z$ is calculated within a given emission region with radius $R$ and magnetic field strength $B$. We locate the TeV blazars on a plot of $B$ vs. $\Gamma R$ (see Figure 1). Three diagonal short dot lines, from top to bottom, indicate the size and magnetic field 
Table 1. The information of emission region of TeV blazars.

\begin{tabular}{|c|c|c|c|c|c|c|}
\hline Object & $\mathbf{z}$ & Type & $B(G)$ & $R\left(10^{16} \mathrm{~cm}\right)$ & $\Gamma$ & Refs. \\
\hline KUV 00311-1938 & - & HBL & 0.40 & 0.65 & 26 & [48] \\
\hline RGB J0152+017 & 0.08 & HBL & $0.14 \pm 0.13$ & $0.58 \pm 0.55$ & $18.3 \pm 11.5$ & {$[48]-[50]$} \\
\hline 3C66A & 0.41 & IBL & $0.44 \pm 0.48$ & $3.47 \pm 3.28$ & $27.8 \pm 4.7$ & [48] [50] [51] \\
\hline 1ES 0229+220 & 0.14 & HBL & $0.24 \pm 0.34$ & $2.70 \pm 1.12$ & $24.2 \pm 22.3$ & [48] [50] \\
\hline PKS 0301-243 & - & HBL & 0.33 & 1 & 2 & [48] \\
\hline RBS 0413 & 0.19 & HBL & $10.11 \pm 17.23$ & $1.07 \pm 0.55$ & $18.3 \pm 2.9$ & {$[52]$} \\
\hline 1ES 0347-121 & 0.188 & HBL & $0.25 \pm 0.39$ & $3.47 \pm 2.41$ & $26.7 \pm 16.6$ & [48] [50] [53] \\
\hline 1ES 0414+009 & 0.287 & HBL & 0.01 & 10 & 50 & {$[54]$} \\
\hline PKS 0447-439 & - & HBL & $0.24 \pm 0.23$ & $0.98 \pm 0.66$ & $29.5 \pm 13.4$ & [48] [55] \\
\hline 1ES 0502+675 & 0.341 & HBL & $0.40 \pm 0.49$ & 1.0 & $16.5 \pm 4.9$ & [48] [56] \\
\hline PKS 0548-322 & 0.069 & HBL & $0.27 \pm 0.29$ & $1.24 \pm 0.79$ & $13.7 \pm 10.0$ & [48] [50] [57] \\
\hline RX J0648.7+1516 & 0.179 & HBL & $3.41 \pm 5.70$ & $1.06 \pm 0.90$ & $18.3 \pm 2.9$ & [58] \\
\hline RGB J0710+591 & 0.125 & HBL & $0.10 \pm 0.05$ & $1.86 \pm 1.34$ & $18.0 \pm 9.5$ & [48] [50] [56] [59] \\
\hline S5 $0716+714$ & 0.31 & IBL & $0.33 \pm 0.25$ & $0.78 \pm 0.06$ & $34.8 \pm 8.2$ & [48] [50] [60] \\
\hline 1ES 0806+524 & 0.138 & HBL & $0.40 \pm 0.07$ & $1.08 \pm 1.11$ & $18.0 \pm 4.0$ & {$[48][50][61]$} \\
\hline 1RXS J101015.9-311909 & 0.143 & HBL & $0.09 \pm 0.10$ & $3.62 \pm 4.78$ & $30.0 \pm 0.0$ & {$[62]$} \\
\hline 1ES 1011+496 & 0.212 & HBL & $0.30 \pm 0.21$ & $1.20 \pm 0.78$ & $29.3 \pm 12.3$ & [48] [50] [63] [64] \\
\hline 1ES 1101-232 & 0.186 & HBL & $0.33 \pm 0.46$ & $2.15 \pm 2.26$ & $23.3 \pm 9.9$ & [48] [50] [65] [66] \\
\hline Mkn 421 & 0.031 & HBL & $0.10 \pm 0.08$ & $2.64 \pm 2.49$ & $31.3 \pm 19.4$ & [48] [50] [67]-[69] \\
\hline Mkn 180 & 0.045 & HBL & $0.18 \pm 0.12$ & $0.45 \pm 0.52$ & $27.5 \pm 18.1$ & [48] [50] [64] \\
\hline 1ES $1215+303$ & 0.13 & HBL & $0.05 \pm 0.04$ & $1.26 \pm 1.42$ & $45.2 \pm 24.4$ & [48] [70] \\
\hline 1ES 1218+304 & 0.182 & HBL & $0.15 \pm 0.04$ & $0.86 \pm 1.15$ & $33.0 \pm 12.1$ & {$[48][50][71]$} \\
\hline W Comae & 0.102 & IBL & $0.20 \pm 0.11$ & $1.92 \pm 2.08$ & $19.7 \pm 4.2$ & [48] [50] [72] [73] \\
\hline $4 C+21.35$ & 0.432 & FSRQ & 0.28 & 0.09 & 75 & {$[74]$} \\
\hline 3C279 & 0.536 & FSRQ & $0.48 \pm 0.62$ & $18.47 \pm 26.28$ & $19.8 \pm 3.8$ & [22] [75]-[77] \\
\hline PKS 1424+240 & - & IBL & $0.28 \pm 0.12$ & $3.42 \pm 1.70$ & $32.9 \pm 13.7$ & [48] [50] [78] \\
\hline H $1426+428$ & 0.129 & HBL & $0.07 \pm 0.05$ & $1.92 \pm 1.05$ & $19.5 \pm 7.2$ & [48] [50] [79] [80] \\
\hline PKS 1510-089 & 0.36 & FSRQ & $0.23 \pm 0.09$ & $6.29 \pm 2.03$ & $15.8 \pm 4.9$ & [81] \\
\hline AP Lib & 0.049 & LBL & 0.01 & 1 & 40 & {$[48]$} \\
\hline PG 1553+113 & 0.5 & HBL & $0.76+0.84$ & $2.26 \pm 2.75$ & $27.5 \pm 7.1$ & [48] [50] [82] \\
\hline Mkn 501 & 0.034 & HBL & $0.25 \pm 0.22$ & $1.31 \pm 2.99$ & $17.6 \pm 5.1$ & [48] [50] [83]-[90] \\
\hline 1ES 1959+650 & 0.048 & HBL & $0.30 \pm 0.38$ & $0.84 \pm 0.45$ & $17 \pm 3.87$ & [48] [50] [91] [92] \\
\hline PKS 2005-489 & 0.071 & HBL & $0.26 \pm 0.31$ & $11.61 \pm 18.98$ & $23.3 \pm 13.0$ & [48] [50] [93] \\
\hline PKS 2155-304 & 0.116 & HBL & $8.24 \pm 25.21$ & $1.31 \pm 1.70$ & $37.9 \pm 27.3$ & $\begin{array}{l}{[48][50][68]} \\
{[77][94][95]}\end{array}$ \\
\hline BL Lacertae & 0.069 & IBL & $0.62 \pm 0.54$ & $0.53 \pm 0.23$ & $16.5 \pm 3.7$ & [48] [50] [96] \\
\hline B3 2247+381 & 0.119 & HBL & $0.07 \pm 0.01$ & $0.60 \pm 0.28$ & $32.5 \pm 3.5$ & [97] \\
\hline 1ES 2344+514 & 0.044 & HBL & $0.09 \pm 0.02$ & $0.75 \pm 0.39$ & $19.0 \pm 6.2$ & [48] [50] [64] [98]-[100] \\
\hline Н 2356-309 & 0.165 & HBL & $0.18 \pm 0.15$ & $0.74 \pm 0.46$ & $24.8 \pm 17.1$ & [48] [50] [77] [101] \\
\hline
\end{tabular}




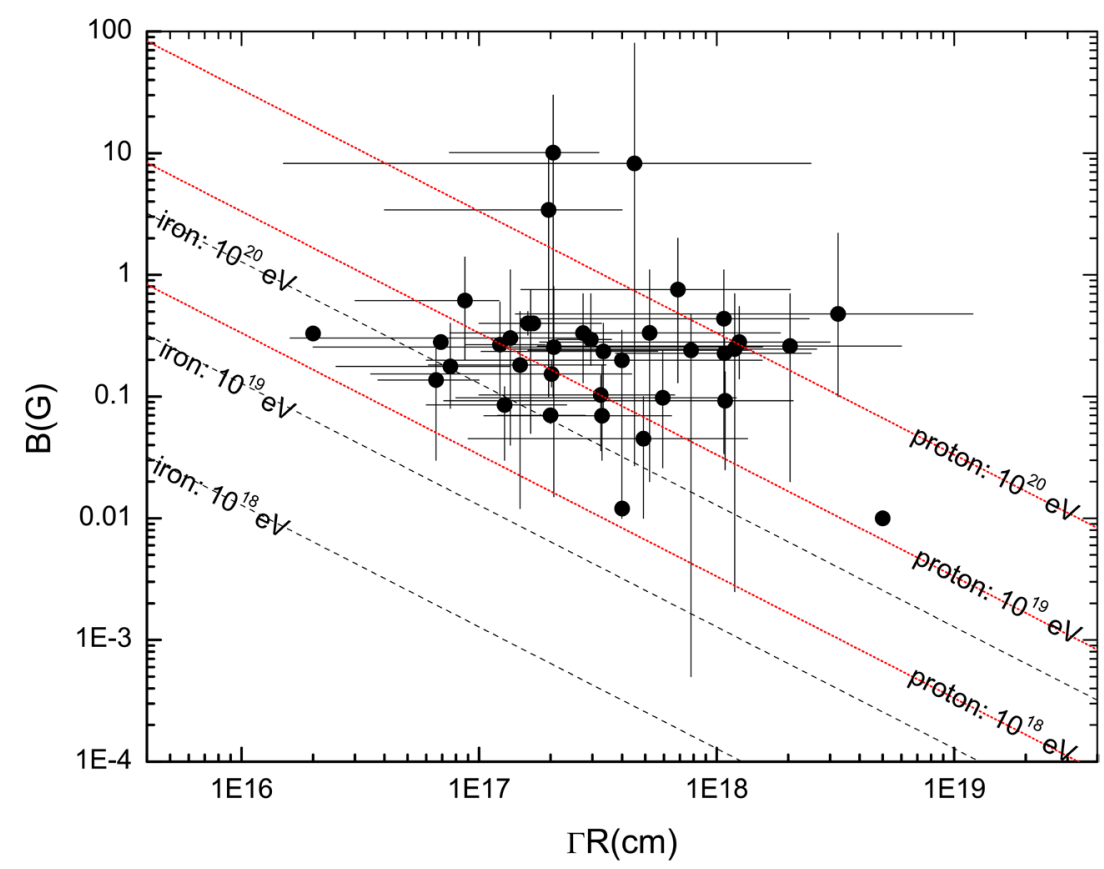

Figure 1. The magnetic field strength vs. the size multiplied by the doppler factor of the emission region for $38 \mathrm{TeV}$ blazars.

strength required of an object to accelerate protons to $10^{20} \mathrm{eV}, 10^{19} \mathrm{eV}$ and $10^{18} \mathrm{eV}$, respectively. Objects below the lines are not up to the corresponding task. The dash lines represent that the object can accelerate irons to $10^{20}$ $\mathrm{eV}, 10^{19} \mathrm{eV}$ and $10^{18} \mathrm{eV}$, respectively. From the figure, one can see that most of the TeV blazars can accelerate the protons to $10^{18} \mathrm{eV}$, but less of them can accelerate protons to $10^{20} \mathrm{eV}$. One can also see that most of the TeV blazars are above the dash lines. This means that they have abilities to accelerate heavy nuclei irons above $10^{20}$ $\mathrm{eV}$. So, TeV blazars can be taken as the candidate for UHECR origin. It should be noted that the acceleration efficiency and the possible energy loss mechanisms when particles are accelerating are not taken into account. It is expected that cosmic ray energy will be degraded by protonpion production on the intense nearby radiation and by synchrotron losses in the associated magnetic fields [24] [29].

\section{Discussion}

As can be seen from Figure 1, most of the TeV blazars can accelerate protons and irons to the energies above $10^{18} \mathrm{eV}$. So, the TeV blazars meet the basic requirement of UHECR sources and can be taken as the sources of UHECRs. Dermer and Razzaque [18] used Fermi data to constrain the maximum energies of cosmic rays accelerated in colliding shells of gamma-ray bursts and blazars. Their results show that irons rather than protons are more likely to be accelerated to ultra high energies in AGNs, and BL Lac objects radiate a volume- and time-averaged emissivity of $10^{45}-10^{46} \mathrm{erg} \mathrm{Mpc}^{-3} \cdot \mathrm{yr}^{-1}$ innonthermal gamma rays. So, BLLac objects have sufficient emissivity to power UHECRs, which require $\sim 10^{44} \mathrm{erg} \mathrm{Mpc}^{-3} \cdot \mathrm{yr}^{-1}$ [30].

The direct way to identify the sources of ultra high energy cosmic rays is to observe their arrival directions, pointing back toward their sources. So, the search for anisotropies in the arrival direction of cosmic rays on different angular scales can contribute to the understanding of the cosmic ray origin, in particular the identification of source regions or individual sources. The correlation between the UHECR arrival directions and the astrophysical objects has been studied by several authors [31]-[38]. However, the claimed correlations between ultra high energy cosmic ray arrival directions and AGNs are controversial.

The largest experiment of Pierre Auger Observatory claimed correlations of UHECRs with the nearby AGNs (redshift $\mathrm{z} \leq$ 0.018) [39] [40] which were not confirmed by HiRes experiment in the Northern hemisphere [41]. The Auger collaboration updated the analysis and found that a smaller fraction of the UHECR events correlate with the same set of AGNs in the latest UHECR data set [42] than in the original one. Using the same parameters reported by the Auger collaboration, Abu-Zayyad et al. searched for the correlation of UHECRs detected by 
the Telescope Array experiment with nearby active galactic nuclei, and found no statistically significant correlation [43].

When searching for potential sources of cosmic rays, the basic obstacle is that charged particles will inevitably deflect as they travel through the magnetic fields that permeate galactic and extragalactic space. The information about their source direction is erased, and this results in their observed arrival directions being almost isotropically distributed. Subsequently, the cosmic ray arrival directions do not point back to any of their powerful candidate sources. After a charged particle travels over a distance $d$ in an extragalactic magnetic field $B_{\text {eg }} \sim$ nG of coherence length $l_{c} \sim 1 \mathrm{Mpc}$, the deflection angle can be estimated as [25]

$$
\theta \sim 1.3^{\circ} \mathrm{Z}\left(\frac{E}{6 \times 10^{19} \mathrm{eV}}\right)^{-1}\left(\frac{d}{10 \mathrm{Mpc}}\right)^{1 / 2}\left(\frac{l_{c}}{\mathrm{Mpc}}\right)^{1 / 2}\left(\frac{B_{e g}}{\mathrm{nG}}\right)
$$

At sufficiently high energies, the deflection is small. This means, for example, that a $10^{20} \mathrm{eV}$ proton propagating through a $1 \mathrm{nG}$ field with a $1 \mathrm{Mpc}$ coherence length (perhaps typical of the extra-galactic field), arriving from $100 \mathrm{Mpc}$, will be deflected by $2.5^{\circ}$ on average. While for a proton with energy of $10^{19} \mathrm{eV}$, the deflection can reach $25^{\circ}$. If for irons, the deflection will be more serious. The galactic magnetic field, locally observed to be on the order of a few $\mu \mathrm{G}$, is also sufficiently strong to induce deflections in the arrival directions of ultra high energy cosmic rays. For a particle traveling a distance $S$ through a uniform magnetic field with perpendicular component $\mathrm{B}$, the angular deflection $\delta$, from its initial trajectory is [44]:

$$
\delta=0.5^{\circ} \mathrm{Z}\left(\frac{\mathrm{S}}{\mathrm{kpC}}\right)\left(\frac{B}{\mu \mathrm{G}}\right)\left(\frac{10^{20} \mathrm{eV}}{E}\right)
$$

the galactic magnetic field strength is $\mu \mathrm{G}$, and the solar system is $\sim 8 \mathrm{kpc}$ from the galactic center. Therefore at energies of $10^{20} \mathrm{eV}$ a proton will experience a deflection of $4^{\circ}$ during propagation.

However, protons with energies of $10^{19} \mathrm{eV}$ will experience larger deflections. This deflection can be sufficiently large as to make source identification difficult. Extragalactic and galactic magnetic fields certainly play an important role in UHECRs propagation. Yet the lack of knowledge concerning the distribution and strength of these fields, at both galactic and extragalactic scales, introduces a large degree of uncertainty in the subject of charged particle propagation [45].

Heavy nuclei have higher electric charge than protons and consequently suffer bigger deflections by magnetic fields. Determining the type of particles that are arriving at earth will also provide indications as to which astrophysical processes could have produced them. A satisfactory understanding of the origin of cosmic rays must deal with the issue of chemical composition. With existing observation techniques, UHECR composition cannot be determined on an event-by-even basis. Only the trends or changes in composition can be measured from the statistics of many events. However, chemical compositions of ultra high energy cosmic rays measured by two largest detectors, High Resolution Fly's Eye (HiRes) and Pierre Auger Observatory (PAO), particularly at the highest energies, are significantly differently. The HiRes data show pure proton composition [46]. On the contrary, the PAO data strongly favor the nuclei composition getting progressively heavier at $E \sim(4-40) \mathrm{EeV}$ [47].

\section{Conclusion}

The origin of UHECRs is one of the most puzzling problems in cosmic ray physics. Astrophysical extragalactic models for the observed UHECR must satisfy some constraints. A minimum requirement for astrophysical sources of UHECRs is the ability to magnetically confine particles of the requisite energies. The results show that $\mathrm{TeV}$ blazars meet this basic criterion, and they can accelerate protons and irons up to ultra high energies. So they can be the potential UHECR sources. However, the detail of the acceleration mechanism is poorly known. In addition, there are some great uncertainties in intervening magnetic fields and cosmic ray primary composition. These cause the lack of any confirmed anisotropy in arrival directions to date, and then hinder the development of theories of cosmic ray origins. Reduction of the uncertainties by large exposure observatories will be one way to unveil these extremely energetic sources. It should be noted that due to interactions between cosmic rays and the CMB protons, UHECR with energies above GZK energy can not be observed on earth when they traveled over distance larger than the GZK horizon ( 100 Mpc). The other messengers, such as neutrinos and high energy gamma rays will also play an important role in identifying the sources of UHECRs. 


\section{Acknowledgements}

This work is supported by the National Natural Science Foundation of China (Grant Nos. 11273008 and 11147134).

\section{References}

[1] Nagano, M., Hara, T., Hatano, Y., et al. (1984) Energy Spectrum of Primary Cosmic Rays between $10^{14.5}$ and $10^{18} \mathrm{eV}$. Journal of Physics G: Nuclear Physics, 10, 1295-1310. http://dx.doi.org/10.1088/0305-4616/10/9/016

[2] Abu-Zayyad, T., Belov, K., Bird, D.J., et al. (2001) Measurement of the Cosmic-Ray Energy Spectrum and Composition from $10^{17}$ to $10^{18.3} \mathrm{eV}$ Using a Hybrid Technique. The Astrophysical Journal, 557, 686-699. http://dx.doi.org/10.1086/322240

[3] Abbasi, R.U., Abu-Zayyad, T., Amann, J.F., et al. (2004) Measurement of the Flux of Ultrahigh Energy Cosmic Rays from Monocular Observations by the High Resolution Fly’s Eye Experiment. Physical Review Letters, 92, Article ID: 151101. http://dx.doi.org/10.1103/PhysRevLett.92.151101

[4] Abraham, J., Abreu, P., Aglietta, M., et al. (2008) Observation of the Suppression of the Flux of Cosmic Rays above $4 \times 10^{19}$ eV. Physical Review Letters, 101, Article ID: 061101. http://dx.doi.org/10.1103/PhysRevLett.101.061101

[5] Greisen K. (1966) End to the Cosmic-Ray Spectrum? Physical Review Letters, 16, 748-750. http://dx.doi.org/10.1103/PhysRevLett.16.748

[6] Zatsepin, G.T. and Kuz’Min, V.A. (1966) Upper Limit of the Spectrum of Cosmic Rays. JETP Letters, 4, 78-80.

[7] Abbasi, R.U., Abu-Zayyad, T., Allen, M., et al. (2008) First Observation of the Greisen-Zatsepin-Kuzmin Suppression. Physical Review Letters, 100, Article ID: 101101. http://dx.doi.org/10.1103/PhysRevLett.100.101101

[8] Allard, D., Parizot, E. and Olinto, A.V. (2007) On the Transition from Galactic to Extragalactic Cosmic-Rays: Spectral and Composition Features from Two Opposite Scenarios. Astroparticle Physics, 27, 61-75. http://dx.doi.org/10.1016/j.astropartphys.2006.09.006

[9] Berezinskii, V.S. and Grigor'eva, S.I. (1988) A Bump in the Ultra-High Energy Cosmic Ray Spectrum. Astronomy \& Astrophysics, 199, 1-12.

[10] Berezinsky, V., Gazizov, A.Z. and Grigoreva, S.I. (2005) Dip in UHECR Spectrum as Signature of Proton Interaction with CMB. Physics Letters B, 612, 147-153. http://dx.doi.org/10.1016/j.physletb.2005.02.058

[11] Nagano, M. and Watson, A.A. (2000) Observations and Implications of the Ultrahigh-Energy Cosmic Rays. Reviews of Modern Physics, 72, 689-732. http://dx.doi.org/10.1103/RevModPhys.72.689

[12] Linsley, J. (1963) Evidence for a Primary Cosmic-Ray Particle with Energy $10^{20}$ eV. Physical Review Letters, 10, 146148. http://dx.doi.org/10.1103/PhysRevLett.10.146

[13] Chiba, N., Hashimoto, K., Hayashida, N., Honda, K., Honda, M., Inoue, N., et al. (1992) Akeno Giant Air Shower Array (AGASA) Covering $100 \mathrm{~km}^{2}$ Area. Nuclear Instruments and Methods in Physics Research Section A: Accelerators, Spectrometers, Detectors and Associated Equipment, 311, 338-349. http://dx.doi.org/10.1016/0168-9002(92)90882-5

[14] Boyer, J.H., Knapp, B.C., Mannel, E.J. and Seman, M. (2002) FADC-Based DAQ for HiRes Fly's Eye. Nuclear Instruments and Methods in Physics Research Section A: Accelerators, Spectrometers, Detectors and Associated Equipment, 482, 457-474. http://dx.doi.org/10.1016/S0168-9002(01)01517-0

[15] Abraham, J., Aglietta, M., Aguirre, I.C., Albrow, M., Allard, D., Allekotte, I., et al. (2004) Properties and Performance of the Prototype Instrument for the Pierre Auger Observatory. Nuclear Instruments and Methods in Physics Research Section A: Accelerators, Spectrometers, Detectors and Associated Equipment, 523, 50-95. http://dx.doi.org/10.1016/j.nima.2003.12.012

[16] Nonaka, T., Abu-Zayyad, T., Allen, M., Azuma, R., Belz, J.W., Bergman, D.R., et al. (2009) The Present Status of the Telescope Array Experiment. Nuclear Physics B, Proceedings Supplements, 190, 26-31. http://dx.doi.org/10.1016/j.nuclphysbps.2009.03.064

[17] Torres, D.F. and Anchordoqui, L.A. (2004) Astrophysical Origins of Ultrahigh Energy Cosmic Rays. Reports on Progress in Physics, 67, 1663-1730.

[18] Dermer, C.D. and Razzaque, S. (2010) Acceleration of Ultra-High-Energy Cosmic Rays in the Colliding Shells of Blazars and Gamma-Ray Bursts: Constraints from the Fermi Gamma-Ray Space Telescope. The Astrophysical Journal, 724, 1366-1372.

[19] Berezinsky, V. (2009) Ultra High Energy Cosmic Ray Protons: Signatures and Observations. Nuclear Physics B, Proceedings Supplements, 188, 227-232. http://dx.doi.org/10.1016/j.nuclphysbps.2009.02.054

[20] Urry, C.M. and Padovani, P. (1995) Unified Schemes for Radio-Loud Active Galactic Nuclei. Publications of the Astronomical Society of the Pacific, 107, 803-845. 
[21] Punch, M., Akerlof, C.W., Cawley, M.F., Chantell, M., Fegan, D.J., Fennell, S., et al. (1992) Detection of TeV Photons from the Active Galaxy Markarian 421. Nature, 358, 477-478. http://dx.doi.org/10.1038/358477a0

[22] Sahayanathan, S. and Godambe, S. (2012) Modelling the Very High Energy Flare of 3C 279 Using One-Zone Leptonic Model. Monthly Notices of the Royal Astronomical Society, 419, 1660-1666. http://dx.doi.org/10.1111/j.1365-2966.2011.19829.x

[23] Bhattacharjee, P. and Sigl, G. (2000) Origin and Propagation of Extremely High-Energy Cosmic Rays. Physics Reports, 327, 109-247. http://dx.doi.org/10.1016/S0370-1573(99)00101-5

[24] Hillas, A.M. (1984) The Origin of Ultra-High-Energy Cosmic Rays. Annual Review of Astronomy and Astrophysics, 22, 425-444. http://dx.doi.org/10.1146/annurev.aa.22.090184.002233

[25] Sigl, G. (2009) Time Structure and Multi-Messenger Signatures of Ultra-High Energy Cosmic Ray Sources. New Journal of Physics, 11, Article ID: 065014.

[26] Halzen, F. and Zas, E. (1997) Neutrino Fluxes from Active Galaxies: A Model-Independent Estimate. The Astrophysical Journal, 488, 669-674.

[27] Stanev, T. (2010) Ultra High Energy Cosmic Rays: Origin and Propagation. Modern Physics Letters A, 25, $1467-1481$. http://dx.doi.org/10.1142/S0217732310033530

[28] Murase, K., Dermer, C.D., Takami, H. and Migliori, G. (2012) Blazars as Ultra-High-Energy Cosmic-Ray Sources: Implications for TeV Gamma-Ray Observations. The Astrophysical Journal, 749, 63-77.

[29] Vietri, M. (1998) On the Energy of Neutrinos from Gamma-Ray Bursts. The Astrophysical Journal, 507, 40-45.

[30] Berezinsky, V., Gazizov, A. and Grigorieva, S. (2006) On Astrophysical Solution to Ultrahigh Energy Cosmic Rays. Physical Review D, 74, Article ID: 043005. http://dx.doi.org/10.1103/PhysRevD.74.043005

[31] Abbasi, R.U., Abu-Zayyad, T., Amann, J.F., Archbold, G., Belov, K., Belz, J.W., et al. (2006) Search for Cross-Correlations of Ultrahigh-Energy Cosmic Rays with BL Lacertae Objects. The Astrophysical Journal, 636, 680-684.

[32] Gorbunov, D.S., Tinyakov, P.G., Tkachev, I.I. and Troitsky, S.V. (2004) Testing the Correlations between UltrahighEnergy Cosmic Rays and BL Lac-Type Objects with HiRes Stereoscopic Data. Journal of Experimental and Theoretical Physics Letters, 80, 145-148. http://dx.doi.org/10.1134/1.1808838

[33] Singh, S., Ma, C.P. and Arons, J. (2004) Gamma-Ray Bursts and Magnetars as Possible Sources of Ultrahigh Energy Cosmic Rays: Correlation of Cosmic Ray Event Positions with IRAS Galaxies. Physical Review D, 69, Article ID: 063003. http://dx.doi.org/10.1103/PhysRevD.69.063003

[34] Smialkowski, A., Giller, M. and Michalak, W. (2002) Luminous Infrared Galaxies as Possible Sources of UHE Cosmic Rays. Journal of Physics G: Nuclear and Particle Physics, 28, 1359-1374.

[35] Torres, D.F., Boldt, E., Hamilton, T. and Loewenstein, M. (2002) Nearby Quasar Remnants and Ultrahigh-Energy Cosmic Rays. Physical Review D, 66, Article ID: 023001. http://dx.doi.org/10.1103/PhysRevD.66.023001

[36] Tinyakov, P.G. and Tkachev, I.I. (2001) BL Lacertae Are Probable Sources of the Observed Ultrahigh Energy Cosmic Rays. Journal of Experimental and Theoretical Physics Letters, 74, 445-448. http://dx.doi.org/10.1134/1.1434282

[37] Tinyakov, P.G. and Tkachev, I.I. (2002) Tracing Protons through the Galactic Magnetic Field: A Clue for Charge Composition of Ultra-High-Energy Cosmic Rays. Astroparticle Physics, 18, 165-172. http://dx.doi.org/10.1016/S0927-6505(02)00109-3

[38] Gorbunov, D.S., Tinyakov, P.G., Tkachev, I.I. and Troitsky, S. (2002) Evidence for a Connection between the Gamma-Ray and the Highest Energy Cosmic-Ray Emissions by BL Lacertae Objects. Astrophysical Journal Letters, 577, 93-96.

[39] Abraham, J., Abreu, P., Aglietta, M., Aguirre, C., Allard, D., Allekotte, I., et al. (2007) Correlation of the HighestEnergy Cosmic Rays with Nearby Extragalactic Objects. Science, 318, 938-943. http://dx.doi.org/10.1126/science.1151124

[40] Abraham, J., Abreu, P., Aglietta, M., Aguirre, C., Allard, D., Allekotte, I., et al. (2008) Correlation of the HighestEnergy Cosmic Rays with the Positions of Nearby Active Galactic Nuclei. Astroparticle Physics, 29, 188-204. http://dx.doi.org/10.1016/j.astropartphys.2008.01.002

[41] Abbasi, R.U., Abu-Zayyad, T., Allen, M., Amman, J.F., Archbold, G., Belov, K., et al. (2008) Search for Correlations between HiRes Stereo Events and Active Galactic Nuclei. Astroparticle Physics, 30, 175-179. http://dx.doi.org/10.1016/j.astropartphys.2008.08.004

[42] Abreu, P., Aglietta, M., Ahn, E.J., Allard, D., Allekotte, I., Allen, J., et al. (2010) Update on the Correlation of the Highest Energy Cosmic Rays with Nearby Extragalactic Matter. Astroparticle Physics, 34, 314-326. http://dx.doi.org/10.1016/j.astropartphys.2010.08.010

[43] Abu-Zayyad, T., Aida, R., Allen, M., Anderson, R., Azuma, R., Barcikowski, E., et al. (2012) Search for Anisotropy of Ultrahigh Energy Cosmic Rays with the Telescope Array Experiment. The Astrophysical Journal, 757, 26-36. 
[44] Finley, C.B. (2006) Anisotropy of Arrival Directions of Ultrahigh Energy Cosmic Rays. Ph.D. Thesis, Columbia University, New York, 18.

[45] Kotera, K., Allard, D., Murase, K., Aoi, J., Dubois, Y., Pierog, T., et al. (2009) Propagation of Ultrahigh Energy Nuclei in Clusters of Galaxies: Resulting Composition and Secondary Emissions. The Astrophysical Journal, 707, 370-386.

[46] Abbasi, R.U., Abu-Zayyad, T., Al-Seady, M., Allen, M., Amman, J.F., Anderson, R.J., et al. (2010) Indications of Proton-Dominated Cosmic-Ray Composition above 1.6 EeV. Physical Review Letters, 104, Article ID: 161101. http://dx.doi.org/10.1103/PhysRevLett.104.161101

[47] Abraham, J., Abreu, P., Aglietta, M., Ahn, E.J., Allard, D., Allekotte, I., et al. (2010) Measurement of the Depth of Maximum of Extensive Air Showers above $10^{18} \mathrm{eV}$. Physical Review Letters, 104, Article ID: 091101. http://dx.doi.org/10.1103/PhysRevLett.104.091101

[48] Tavecchio, F., Ghisellini, G., Ghirlanda, G., Foschini, L. and Maraschi, L. (2010) TeV BL Lac Objects at the Dawn of the Fermi Era. Monthly Notices of the Royal Astronomical Society, 401, 1570-1586. http://dx.doi.org/10.1111/j.1365-2966.2009.15784.x

[49] Aharonian, F., Akhperjanian, A.G., De Almeida U.B., Bazer-Bachi, A.R., Behera, B., Beilicke, M., et al. (2008) Discovery of VHE Gamma-Rays from the High-Frequency-Peaked BL Lacertae Object RGB J0152+017. Astronomy and Astrophysics, 481, L103-L107. http://dx.doi.org/10.1051/0004-6361:200809603

[50] Zhang, J., Liang, E.W., Zhang, S.N. and Bai, J.M. (2012) Radiation Mechanisms and Physical Properties of GeV-TeV BL Lac Objects. The Astrophysical Journal, 752, 157. http://dx.doi.org/10.1088/0004-637X/752/2/157

[51] Yang, J. and Wang, J. (2010) Mechanism of Very High-Energy Radiation in BL Lacertae Object 3C 66A. Astronomy and Astrophysics, 511, 11-15. http://dx.doi.org/10.1051/0004-6361/200913035

[52] Aliu, E., Archambault, S., Arlen, T., Aune, T., Beilicke, M., Benbow, W., et al. (2012) Discovery of High-Energy and Very High Energy Gamma-Ray Emission from the Blazar RBS 0413. The Astrophysical Journal, 750, 94-99.

[53] Aharonian, F., Akhperjanian, A.G., De Almeida, U.B., Bazer-Bachi, A.R., Behera, B., Beilicke, M., et al. (2007) Discovery of VHE Gamma-Rays from the Distant BL Lacertae 1ES 0347-121. Astronomy and Astrophysics, 473, 25-28. http://dx.doi.org/10.1051/0004-6361:20078412

[54] Abramowski, A., Acero, F., Aharonian, F., Akhperjanian, A.G., Anton, G., Balzer, A., et al. (2012) Discovery of HardSpectrum $\gamma$-Ray Emission from the BL Lacertae Object 1ES 0414+009. Astronomy and Astrophysics, 538, 103-111. http://dx.doi.org/10.1051/0004-6361/201118406

[55] Prandini, E., Bonnoli, G. and Tavecchio, F. (2012) Estimating the Redshift of PKS 0447-439 through Its GeV-TeV Emission. Astronomy and Astrophysics, 543, 111-115. http://dx.doi.org/10.1051/0004-6361/201118289

[56] Katarzynski, K. (2012) Hard MeV-GeV Spectra of Blazars. Astronomy and Astrophysics, 537, 47-55. http://dx.doi.org/10.1051/0004-6361/201116839

[57] Aharonian, F., Akhperjanian, A.G., Anton, G., de Almeida, U.B., Bazer-Bachi, A.R., Becherini, Y., et al. (2010) Discovery of VHE $\gamma$-Rays from the BL Lacertae Object PKS 0548-322. Astronomy and Astrophysics, 521, 69-74. http://dx.doi.org/10.1051/0004-6361/200912363

[58] Aliu, E., Aune, T., Beilicke, M., Böttcher, M., Bouvier, A., Bradbury, S.M., et al. (2011) Multiwavelength Observations of the Previously Unidentified Blazar RX J0648.7+1516. The Astrophysical Journal, 742, 127-133.

[59] Acciari, V.A., Aliu, E., Arlen, T., Aune, T., Bautista, M., Beilicke, M., et al. (2010) The Discovery of Gamma-Ray Emission from the Blazar RGB J0710+591. Astrophysical Journal Letters, 715, 49-55.

[60] Anderhub, H. (2009) Discovery of Very High Energy Gamma-Rays from the Blazar S5 0716+714. Astrophysical Journal Letters, 704, 129-133.

[61] Acciari, V., Aliu, E., Arlen, T., Bautista, M., Beilicke, M., Benbow, W., et al. (2009) Discovery of Very High Energy Gamma-Ray Radiation from the BL Lac 1ES 0806+524. Astrophysical Journal Letters, 690, 126-129.

[62] Abramowski, A., Acero, F., Aharonian, F., Akhperjanian, A.G., Anton, G., Balzer, A., et al. (2012) Discovery of VHE Gamma-Ray Emission and Multi-Wavelength Observations of the BL Lacertae Object 1RXS J101015.9-311909. Astronomy and Astrophysics, 542, 94-103. http://dx.doi.org/10.1051/0004-6361/201218910

[63] Reinthal, R., Rugamer, S., Lindfors, E.J., Mazin, D., Stamerra, A., Longo, F., et al. (2012) Multi-Wavelength Observations of the HBL 1ES 1011+496 in Spring 2008. Journal of Physics: Conference Series, 355, Article ID: 012017.

[64] Rugamer, S., Angelakis, E., Bastieri, D., Dorner, D., Kovalev, Y.Y., Lähteenmäki, A., et al. (2008) MAGIC and MultiWavelength Observations of Three HBLs in 2008.

[65] Aharonian, F., Akhperjanian, A.G., Bazer-Bachi, A.R., Beilicke, M., Benbow, W., Berge, D., et al. (2007) Detection of VHE Gamma-Ray Emission from the Distant Blazar 1ES 1101-232 with HESS and Broadband Characterisation. Astronomy and Astrophysics, 470, 475-489. http://dx.doi.org/10.1051/0004-6361:20077057 
[66] Yan, D., Zeng, H. and Zhang, L. (2012) Non-Variable TeV Emission from the Extended Jet of a Blazar in the Stochastic Acceleration Scenario: The Case of the Hard TeV Emission of 1ES 1101-232. Monthly Notices of the Royal Astronomical Society, 424, 2173-2179. http://dx.doi.org/10.1111/j.1365-2966.2012.21376.X

[67] Acciari, V.A., Aliu, E., Arlen, T., Aune, T., Beilicke, M., Benbow, W., et al. (2011) TeV and Multi-Wavelength Observations of Mrk 421 in 2006-2008. The Astrophysical Journal, 738, 25-43.

[68] Finke, J.D., Dermer, C.D. and Bottcher, M. (2008) Synchrotron Self-Compton Analysis of TeV X-Ray-Selected BL Lacertae Objects. The Astrophysical Journal, 686, 181-194.

[69] Shukla, A., Chitnis, V.R., Vishwanath, P.R., Acharya, B.S., Anupama, G.C., Bhattacharjee, P., et al. (2012) Multiwavelength Study of the TeV Blazar Mrk 421 during a Giant Flare. Astronomy and Astrophysics, 541, 140-148. http://dx.doi.org/10.1051/0004-6361/201118569

[70] Aleksic, J., Alvarez, E.A., Antonelli, L.A., Antoranz, P., Ansoldi, S., Asensio, M., et al. (2012) Discovery of VHE Gamma-Rays from the Blazar 1ES 1215+303 with the MAGIC Telescopes and Simultaneous Multi-Wavelength Observations. Astronomy and Astrophysics, 544, 142-151. http://dx.doi.org/10.1051/0004-6361/201219133

[71] Weidinger, M. and Spanier, F. (2010) Modelling the Variability of 1ES1218+30.4. Astronomy and Astrophysics, 515, 18-23. http://dx.doi.org/10.1051/0004-6361/201014299

[72] Acciari, V.A., Aliu, E., Aune, T., Beilicke, M., Benbow, W., Bottcher, M., et al. (2009) Multiwavelength Observations of a TeV-Flare from W Comae. The Astrophysical Journal, 707, 612-620.

[73] Zhang, J. (2009) A Synchrotron Self-Compton Scenario for the Very High Energy Gamma-Ray Emission of the Intermediate BL Lacertae Object W Comae. Research in Astronomy and Astrophysics, 9, 777-782.

[74] Tavecchio, F., Becerra-Gonzalez, J., Ghisellini, G., Stamerra, A., Bonnoli, G., Foschini, L., et al. (2011) On the Origin of the Gamma-Ray Emission from the Flaring Blazar PKS 1222+216. Astronomy and Astrophysics, 534, 86-93. http://dx.doi.org/10.1051/0004-6361/201117204

[75] Aleksic, J., Antonelli, L.A., Antoranz, P., Backes, M., Barrio, J.A., Bastieri, D., et al. (2011) MAGIC Observations and Multiwavelength Properties of the Quasar 3C 279 in 2007 and 2009. Astronomy and Astrophysics, 530, 4-14. http://dx.doi.org/10.1051/0004-6361/201116497

[76] Hayashida, M., Madejski, G.M., Nalewajko, K., Sikora, M., Wehrle, A.E., Ogle, P., et al. (2012) The Structure and Emission Model of the Relativistic Jet in the Quasar 3C 279 Inferred from Radio to High-Energy Gamma-Ray Observations in 2008-2010. The Astrophysical Journal, 754, 114-135.

[77] Mizumura, Y., Kushida, J., Nishijima, K., Bicknell, G.V., Clay, R.W., Edwards, P.G., et al. (2012) Searches for Very High Energy gamma Rays from Blazars with CANGAROO-III Telescope in 2005-2009. Astroparticle Physics, 35, 563-572. http://dx.doi.org/10.1016/j.astropartphys.2012.01.002

[78] Acciari, V.A., Aliu, E., Arlen, T., Aune, T., Bautista, M., Cogan, P., et al. (2010) Discovery of Very High Energy Gamma Rays from PKS 1424+240 and Multiwavelength Constraints on Its Redshift. Astrophysical Journal Letters, 708, 100-106.

[79] Costamante, L., Aharonian, F., Ghisellini, G. and Horns, D. (2003) The SED of the TeV BLLac 1ES 1426+428 after Correction for the TeV-IR Absorption. New Astronomy Reviews, 47, 677-680. http://dx.doi.org/10.1016/S1387-6473(03)00121-0

[80] Kato, T., Kusunose, M. and Takahara, F. (2006) Constraining the Emission Properties of TeV Blazar H1426+428 by the Synchrotron Self-Compton Model. The Astrophysical Journal, 638, 653-658.

[81] Chen, X., Fossati, G., Böttcher, M. andLiang, E. (2012) Time-Dependent Simulations of Emission from the FSRQ PKS 1510-089: Multiwavelength Variability of External Compton and Synchrotron Self-Compton Models. Monthly Notices of the Royal Astronomical Society, 424, 789-799. http://dx.doi.org/10.1111/j.1365-2966.2012.21283.x

[82] Aleksi, C.J., Alvarez, E.A., Antonelli, L.A., Antoranz, P., Asensio, M., Backes, M., et al. (2012) PG 1553+113: Five Years of Observations with MAGIC. The Astrophysical Journal, 748, 46-55.

[83] Abdo, A.A., Ackermann, M., Ajello, M., Allafort, A., Aleksić, J., Acciari, V.A., et al. (2011) Insights into the HighEnergy Gamma-Ray Emission of Markarian 501 from Extensive Multifrequency Observations in the Fermi Era. The Astrophysical Journal, 727, 129-154.

[84] Acciari, V.A., Arlen, T., Aune, T., Beilicke, M., Benbow, W., Böttcher, M., et al. (2011) Spectral Energy Distribution of Markarian 501: Quiescent State versus Extreme Outburst. The Astrophysical Journal, 729, 2-10.

[85] Albert, J., Aliu, E., Anderhub, H., Antoranz, P., Armada, A., Baixeras, C., et al. (2007) Variable Very High Energy Gamma-Ray Emission from Markarian 501. The Astrophysical Journal, 669, 862-883.

[86] Anderhub, H., Antonelli, L.A., Antoranz, P., Sato, R., Ushio, M., Kataoka, J., et al. (2009) Simultaneous Multiwavelength Observation of Mkn 501 in a Low State in 2006. The Astrophysical Journal, 705, 1624-1631. 
[87] Katarzynski, K., Sol, H. and Kus, A. (2001) The Multifrequency Emission of Mrk 501. From Radio to TeV GammaRays. Astronomy and Astrophysics, 367, 809-825. http://dx.doi.org/10.1051/0004-6361:20000538

[88] Petry, D., Bottcher, M., Connaughton, V., Lahteenmaki, A., Pursimo, T., Raiteri, C.M., et al. (2000) Multiwavelength Observations of Markarian 501 during the 1997 High State. The Astrophysical Journal, 536, 742-755.

[89] Pian, E., Vacanti, G., Tagliaferri, G., Ghisellini, G., Maraschi, L., Treves, A., et al. (1998) BeppoSAX Observations of Unprecedented Synchrotron Activity in the BL Lacertae Object Markarian 501. Astrophysical Journal Letters, 492, 1720.

[90] Sambruna, R.M., Aharonian, F.A., Krawczynski, H., Akhperjanian, A.G., Barrio, J.A., Bernlöhr, K., et al. (2000) Correlated Intense X-Ray and TEV Activity of Markarian 501 in 1998 June. The Astrophysical Journal, 538, 127-133.

[91] Krawczynski, H., Hughes, S.B., Horan, D., Aharonian, F., Aller, M.F., Aller, H., et al. (2004) Multiwavelength Observations of Strong Flares from the TeV Blazar 1ES 1959+650. The Astrophysical Journal, 601, 151-164.

[92] Tagliaferri, G., Foschini, L., Ghisellini, G., Maraschi, L., Tosti, G., Albert, J., et al. (2008) Simultaneous Multiwavelength Observations of the Blazar 1ES 1959+650 at a Low TeV Flux. The Astrophysical Journal, 679, 1029-1039.

[93] Abramowski, A., Acero, F., Aharonian, F., Akhperjanian, A.G., Anton, G., Barnacka, A., et al. (2011) Simultaneous Multi-Wavelength Campaign on PKS 2005-489 in a High State. Astronomy and Astrophysics, 533, 110-118. http://dx.doi.org/10.1051/0004-6361/201016170

[94] Abramowski, A., Acero, F., Aharonian, F., Akhperjanian, A.G., Anton, G., Balzer, A., et al. (2012) A Multiwavelength View of the Flaring State of PKS 2155-304 in 2006. Astronomy and Astrophysics, 539, 149-170. http://dx.doi.org/10.1051/0004-6361/201117509

[95] Cerruti, M., Zech, A., Boisson, C. and Inoue, S. (2011) SF2A-2011: Proceedings of the Annual Meeting of the French Society of Astronomy and Astrophysics. 555-558.

[96] Potter, W.J. and Cotter, G. (2012) Synchrotron and Inverse-Compton Emission from Blazar Jets-I. A Uniform Conical jet Model. Monthly Notices of the Royal Astronomical Society, 423, 756-765. http://dx.doi.org/10.1111/j.1365-2966.2012.20918.x

[97] Aleksic, J., Alvarez, E.A., Antonelli, L.A., Antoranz, P., Asensio, M., Backes, M., et al. (2012) Discovery of VHE Gamma-Ray Emission from the BL Lacertae Object B3 2247+381 with the MAGIC Telescopes. Astronomy and Astrophysics, 539, 118-123. http://dx.doi.org/10.1051/0004-6361/201117967

[98] Acciari, V.A., Aliu, E., Arlen, T., Aune, T., Beilicke, M., Benbow, W., et al. (2011) Multiwavelength Observations of the Very High Energy Blazar 1ES 2344+514. The Astrophysical Journal, 738, 169-176.

[99] Albert, J., Aliu, E., Anderhub, H., Antoranz, P., Armada, A., Baixeras, C., et al. (2007) Observation of Very High Energy gamma-Rays from the AGN 1ES 2344+514 in a Low Emission State with the MAGIC Telescope. The Astrophysical Journal, 662, 892-899.

[100] Aleksic, J., Antonelli, L.A., Antoranz, P., Asensio, M., Backes, M., de Almeida, U.B., et al. (2013) The Simultaneous Low State Spectral Energy Distribution of 1ES 2344+514 from Radio to Very High Energies. Astronomy and Astrophysics, 556, 67-94. http://dx.doi.org/10.1051/0004-6361/201220714

[101] Abramowski, A., Acero, F., Aharonian, F., Akhperjanian, A.G., Anton, G., de Almeida, U.B., et al. (2010) Multi-Wavelength Observations of H 2356-309. Astronomy and Astrophysics, 516, 56-66. http://dx.doi.org/10.1051/0004-6361/201014321 
Scientific Research Publishing (SCIRP) is one of the largest Open Access journal publishers. It is currently publishing more than 200 open access, online, peer-reviewed journals covering a wide range of academic disciplines. SCIRP serves the worldwide academic communities and contributes to the progress and application of science with its publication.

Other selected journals from SCIRP are listed as below. Submit your manuscript to us via either submit@scirp.org or Online Submission Portal.
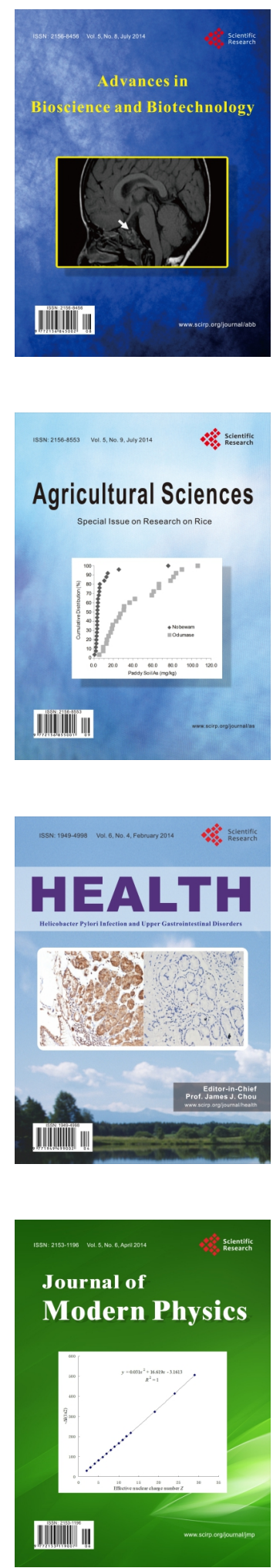
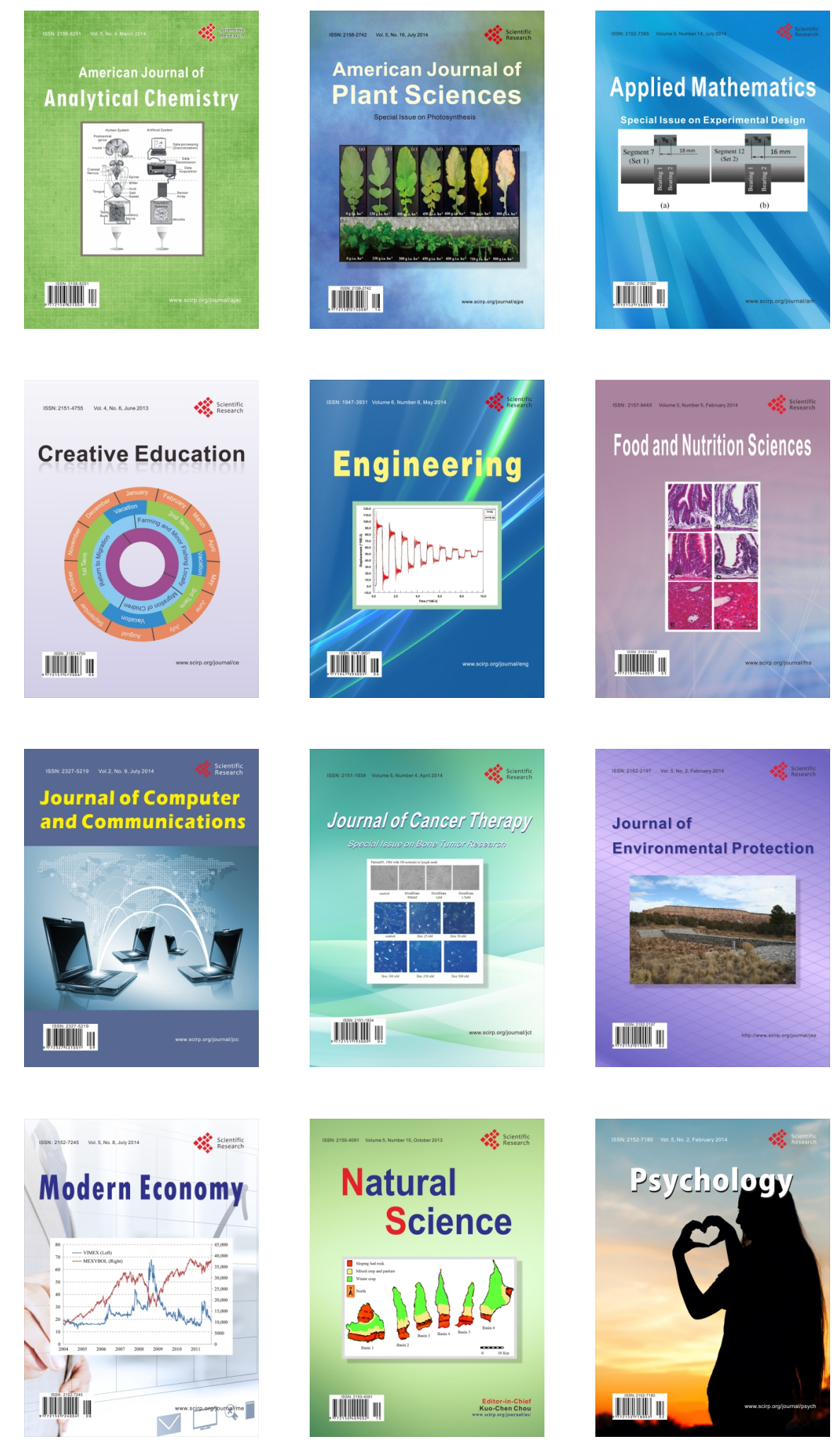